\title{
RECENT RESULTS: COLEIDER DETECTOR AT FERMILAB
}

\author{
The CDF Collaboration ${ }^{\star}$
}

Presented by Virgil E. BARNES

Purdue University, West Lafayette, Indiana 47907

The status and prospects of the 1988-89 CDF data taking run with pp collisions at $1.8 \mathrm{TeV}$, are briefly reviewed. Results are presented on minimum-bias inelastic events from 1987 data at 1.8 and $0.63 \mathrm{TeV}$ center of mass energy, and are compared with data at lower energy and with data from the $\mathrm{C} 0$ experiment Transverse momentum spectra harden as the center of mass energy $\sqrt{s}$ increases. Charged particle density in rapidity, and mean transverse momentum, increase faster than $\ln (\mathrm{s})$. Charged particles frequently form jet-like clusters with transverse energy of a few GeV. The present CDF data do not permit conclusions for or against the possible formation of a quark gluon plasma.

\section{INTRODUCTION}

The Collider Detector at Fermilab is a large, general purpose azimuthally symmetric $\sim 4 \pi$ detector covering polar angles from $2^{\circ}$ to $178^{\circ}$ with respect to the collision axis, corresponding to a range of pseudorapidity $-4<\eta<4[\eta=\ln (\tan (\theta / 2))]$. CDF has high resolution tracking in $\mathrm{a} \sim 1.5 \mathrm{~T}$ magnetic field followed by fine-grained projective tower calorimeters, and then by muon tracking over portions of the above range of $\eta$. The detector has been described in detail elsewhere ${ }^{[1]}$. It is well suited to studying individual charged hadrons, jets, and leptons having a wide range of energies and transverse momenta.

In Section 2, the potential of CDF for studying hard collision processes, production of heavy particles, quark compositeness, and minimum bias inelastic events will be discussed in the context of the ongoing high-statistics data run. Sections 3 and 4 discuss central portions of the detector, and the trigger and event selection for minimum bias events recorded in the first CDF physics run, in 1987. Data will include: (Sect. 5) charged particle multiplicities, $\mathrm{N}$, and rapidity density, $\mathrm{dN} / \mathrm{d} \eta,(6)$ charged particle $p_{T}$ spectra, mean $p_{T}$, and $\left\langle p_{T}\right\rangle$ vs $\mathrm{N},(7)$ the $p_{T}$ spectra of $\mathrm{K}_{s}^{o}$, and (8) charged particle correlations and clustering.

\section{STATUS AND PROSPECTS OF THE 1988-89 RUN}

In September, 1988 the TeVatron collider reached and exceeded the design luminosity of $1.0 \times 10^{30} \mathrm{~cm}^{-2} \mathrm{sec}^{-1}$ in several $6 \times 6$ bunch $p \bar{p}$ stores at $1.8 \mathrm{TeV}$. By the end of the month the collider had delivered over $1 \mathrm{pb}^{-1}$ of time-integrated luminosity to CDF in the $\mathrm{BO}$ collision area, and the average rate had risen to $\sim 1 \mathrm{pb}^{-1} /$ month. This extrapolates to $8 \mathrm{pb}^{-1}$ delivered by the end of the run on April 30, 1989. [By November 1, CDF had recorded on magnetic tape over $770 \mathrm{nb}^{-1}$ of data, and was utilizing the delivered luminosity with good efficiency.] The present goal is to have a data sample greater than $3 \mathrm{pb}^{-1}$ for 1988-89.

* ANL-Brandeis-University of ('hicago- Fermilab-INFN, Frascati-Harvard-University of Illinois-KEK-LBLUniversity of Pennsylvania-INFN, University and Scuole Normale, Pisa-Purdue- Rockefeller-RutgersTexas A\&M-Tsukuba-University of Wisconsin

Talk presented at Quark Matter '88 Conference, Lenox, MA, September 25-30, 1988; published in proceedings. 
At the design luminosity, the event rate seen by the minimum bias inelastic event trigger is $44 \mathrm{kHz}$. About 1.5 events per secônd are selected to be written to tape. A Fastbus-based Level 2 hardware trigger system, followed by a software Level 3 trigger using a 30-node ACP computer farm, accepts more than one dozen trigger categories designed to select electrons or muons with large $p_{T}$, dilepton events, high $p_{T}$ hadronic jets or multiple jets, and events with large missing transverse energy signalling the presence of a neutrino or some other neutral non-interacting particle.

Among a few highlights of the potential of the present run are:

A.) More than $3000 \mathrm{~W}^{ \pm} \rightarrow \mathrm{e} \nu$ events and more than $300 \mathrm{Z} \rightarrow \mathrm{e}^{+} \mathrm{e}^{-}$events, where an electron is seen in the central calorimeters. This is a substantial increase in the world sample of $\mathrm{W}$ and $\mathrm{Z}$. The Standard Model predicts ${ }^{[2]}$ (and CDF observed in the 33nb ${ }^{-1}$ data run in $1987)^{[3]}$ a $\mathrm{W}$ production cross section at $1.8 \mathrm{TeV} \sim 3.6(4.6)$ times greater than at $630 \mathrm{GeV}$.

B.) Although particle production cross sections drop rapidly with increasing particle mass, there is a substantial cross section advantage at $1.8 \mathrm{TeV}$ compared to, say, $630 \mathrm{GeV}$. This advantage increases with particle mass ${ }^{[4]}$, and is a factor of order 100 for particles with $300 \mathrm{GeV} / \mathrm{c}^{2}$ mass. CDF will be able either to observe, or else set mass limits on, hypothetical new $Z^{\prime}$ and $W^{\prime}$ bosons up to masses of 400 and $500 \mathrm{GeV} / \mathrm{c}^{2}$, respectively. In general, searches for new heavy particles predicted, for example, by Supersymmetry or Technicolor theories, or for the Higgs particle, should have sensitivity up to several hundred $\mathrm{GeV}$ masses.

C.) Some $100 t \bar{t}$ pairs would be produced ${ }^{[4]}$ if the top quark mass is $110 \mathrm{GeV} / \mathrm{c}^{2}$. The dominant decay mode would be $t \rightarrow b+W$, with $34 \%$ probability that one of the Ws would decay to $\mathrm{e} \nu$ or $\mu \nu$, modes for which standard model backgrounds to the top signal are less severe. It is estimated that CDF may be sensitive to the top quark up to about $100 \mathrm{GeV}$ mass in the present run.

D.) The Jet $p_{T}$ spectrum will be studied well beyond $300 \mathrm{GeV} / \mathrm{c}$, further improving tests of QCD and/or probing for quark compositeness beyond the limit $\Lambda_{c}>700 \mathrm{GeV}$ established from the $1987 \mathrm{CDF}$ data ${ }^{[5]}$.

E.) A small number of $\mathrm{J} / \psi \rightarrow \mu^{+} \mu^{-}$have been seen in jet trigger events from the 1987 run. A low-mass dimuon trigger in the 1988 run is hoped to accept some $\mathrm{J} / \psi$.

F.) Once substantial integrated luminosity has been acquired for hard collisions, it is hoped to extend the study of minimum bias processes in a variety of ways: (a) implement a Level 3 trigger for high charged particle multiplicities; (b) a Level 3 trigger for decays of neutral strange particles to two charged particles; (c) run with a series of jet triggers having relatively low thresholds in $\Sigma E_{T}$ (scalar sum of calorimeter energies weighted by $\sin (\theta)$ ), ranging from 5 to $25 \mathrm{GeV} / \mathrm{c}$; (d) runs at lower $\sqrt{s}$, such as 630 and $1100 \mathrm{GeV}$; (e) study of elastic and single diffractive dissociation events using the small-angle forward detectors consisting of Silicon microstrip detectors and drift chambers mounted in Roman pots; and (f) runs at somewhat reduced magnetic field to extend the charged particle $p_{T}$ spectrum below $400 \mathrm{MeV} / \mathrm{c}$.

\section{THE CENTRAL TRACKING DETECTORS}

The present minimum bias data analysis uses charged tracks found in the Vertex Time Projection Chamber (VTPC) ${ }^{|6|}$ or in the Central Track Chamber (CTC) ${ }^{[7]}$ (Fig. 1).

The VTPC covers a range $|\eta|<3.5$, with full acceptance for $|\eta|<3.0$. It consists of 8 modules each $30 \mathrm{~cm}$ long in $\mathrm{Z}$ and $22 \mathrm{~cm}$ in radius, with $15 \mathrm{~cm}$ drift distance in each 
half cell. There are 3072 sense wires which lie on the chords of circles centered on the collision axis. Sense wires are arranged in octants. Azimuthal coverage is complete. Tracks are reconstructed in the $\mathrm{R}-\mathrm{Z}$ (non-bending) direction, with resolution $\sigma \sim 200$ to $500 \mu \mathrm{m}$ (depending on $\theta$ ) and two-track resolution $6 \mathrm{~mm}$ at $\theta=90^{\circ}$. Cathode pads installed on some modules provide limited R- $\phi$ tracking, but those data were not used in the present analysis.

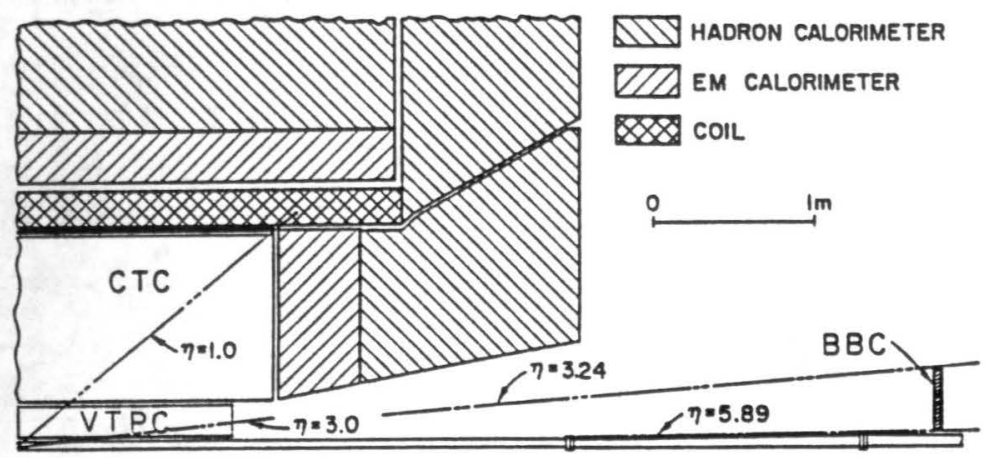

Fig. 1 Sectional view of one-quarter of the central portion of the CDF detector.

The CTC is a large drift chamber with inner radius $0.3 \mathrm{~m}$ and outer radius $1.3 \mathrm{~m}$. Sense wires are arranged in 5 axial superlayers of 12 layers each, and 4 small-angle stereo $\left( \pm 3^{\circ}\right)$ superlayers of 6 layers each, providing full 3-dimensional reconstruction for $|\eta|<\sim$ 1.3. Coverage is complete in azimuth. The transverse momentum resolution, including both single hit resolution and multiple scattering, was $\sigma_{p_{T}} / p_{T}^{2} \leq 0.003 \mathrm{GeV}^{-1}$ for $|\eta| \leq 1.0$. The CTC data permit reconstruction of over 100 tracks in events with high local track density.

\section{TRIGGER AND EVENT SELECTION}

Scintillator hodoscopes located at $\mathrm{Z}$ (along the beam) $= \pm 5.82 \mathrm{~m}$ and covering the range $3.24<|\eta|<5.89$ (the Beam-Beam Counters or BBC) are used to trigger on most of the inelastic cross section, and also serve as a luminosity monitor.

The minimum bias (MB) data samples were obtained with a trigger requiring that at least one charged particle traverse each set of $\mathrm{BBC}$, in coincidence with the beam crossing. At $1.8 \mathrm{TeV}(630 \mathrm{GeV}), 55,700(9,400)$ triggers were analyzed. Beam-beam collision events were selected by requiring that (a) at least 4 charged particles were found in the VTPC in the range $-3<\eta<3$ and at least one of these tracks be in each hemisphere $(\eta>0$ and $\eta<0$ ), and (b) the $\mathrm{Z}$ of the vertex from $\mathrm{BBC}$ timing agreed within $16 \mathrm{~cm}$ with $\mathrm{Z}_{V T P C}$ determined from VTPC tracks.

The Beam-Beam event vertices were distributed gaussianly in $\mathrm{Z}$ with a typical $\sigma_{z}$ of $\mathbf{4 0}$ $\mathrm{cm}$. To ensure uniform VTPC track acceptance, we also required $\left|Z_{V T P C}\right|<65 \mathrm{~cm}$. After these cuts, the contamination of non-beam-beam events was estimated to be less than $2.5 \%$ in the single low-luminosity $630 \mathrm{GeV}$ run, and less than $0.5 \%$ at $1.8 \mathrm{TeV}$, where the typical luminosity was up to $4 \times 10^{28} \mathrm{~cm}^{-2} \mathrm{sec}^{-1}$ for $\mathrm{MB}$ runs. The final $\mathrm{MB}$ sample consists of $51,000(4,800)$ events at the two energies.

The effective $\mathrm{BBC}$ trigger cross section was estimated by extrapolating the total and elastic cross sections to $1.8 \mathrm{TeV}$, giving $75 \mathrm{mb}$ and $15 \mathrm{mb}$, respectively. The $60 \mathrm{mb}$ of 
inelastic cross section is divided into single diffractive (SD), double diffractive (DD), and non diffractive (ND) events. The âcceptances $(\epsilon)$ of the BBC were found by Monte Carlo simulation and are tabulated below:

$$
\begin{array}{|ccccc|}
\hline \sqrt{s}(\mathrm{TeV}) & \epsilon: \mathrm{ND} & \mathrm{DD} & \mathrm{SD} ; & \sigma_{\text {eff }}(\mathrm{mb}) \\
1.8 & 96 \% & 57 \% & 16 \% & 43 \pm 6 \\
0.63 & 93 \% & 57 \% & 11 \% & 34 \pm 3 \\
\hline
\end{array}
$$

A recent UA4 measurement ${ }^{[8]}$ of $\sigma_{S D}=9.4 \pm 0.7 \mathrm{mb}$ at $546 \mathrm{GeV}$ lowers the estimates of $\sigma_{N D}$ and hence lowers both $\sigma_{\text {eff }}$ estimates. Since $\sigma_{D D}$ is a few mb, the CDF MB sample is dominantly non-diffractive inelastic events.

\section{CHARGED PARTICLES: MULTIPLICITY AND DENSITY}

The VTPC counts particles with good efficiency up to $\eta=3$. A correction has been applied for geometrical acceptance as a function of $\eta$ and of $\mathrm{Z}$ of the vertex. Several necessary corrections have not yet been applied: (a) tracks with $p_{T}$ below $50 \mathrm{MeV} / \mathrm{c}$ will not traverse the full radius of the VTPC, and will spiral along $\mathrm{Z}$, resulting in a $5-10 \%$ inefficiency; (b) particle decays, photon conversions, and secondary interactions can add particles which pass the $5 \sigma$ vertex-pointing cut, a $10-15 \%$ effect; (c) recent studies involving Monte Carlo event simulation and track reconstruction, and human scanning with interactive track reconstruction, indicate that track reconstruction inefficiencies and errors are $3-5 \%$.

We estimate that without the above corrections, the overall error ( $\eta$-dependent) is $\sim 10 \%$ or less. These errors are largely common to the $1.8 \mathrm{TeV}$ and $630 \mathrm{GeV}$ data and should cancel when taking ratios.

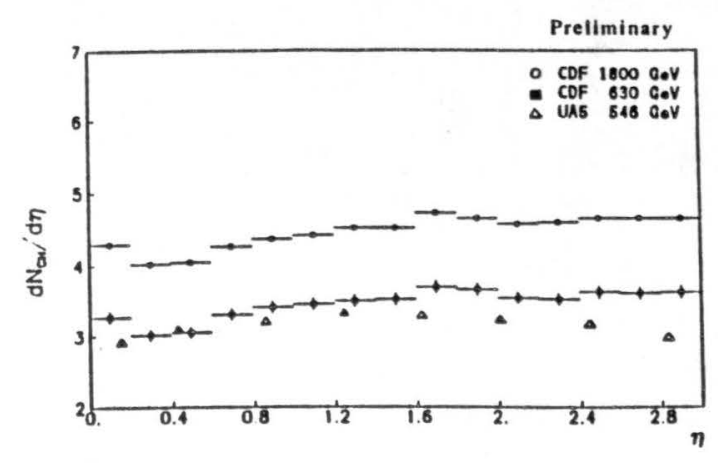

Fig. $2 d N / d \eta \quad$ vs. $\eta \quad$ for CDF data at 0.63 and $1.8 \mathrm{TeV}$, and for UA5 data at $0.546 \mathrm{TeV}$.

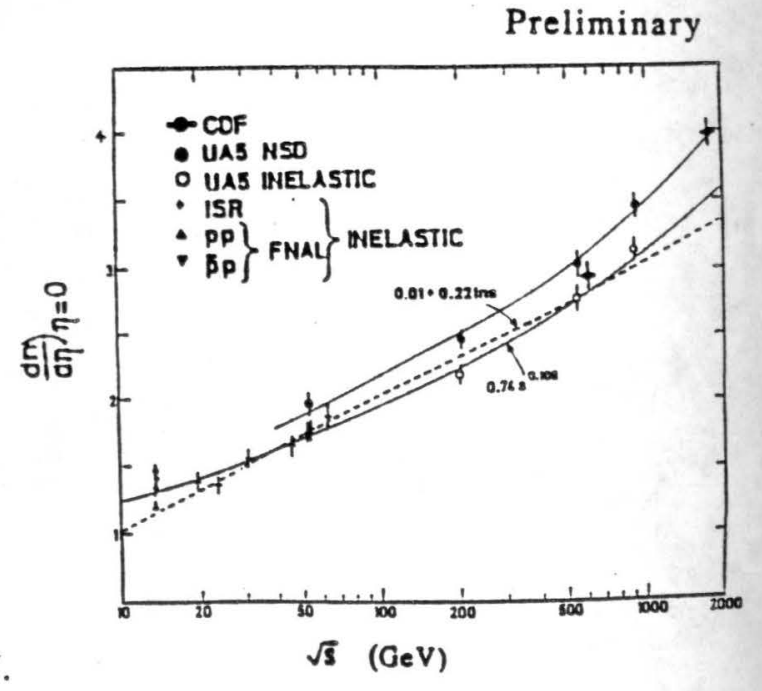

Fig. $3 \mathrm{dN} /\left.\mathrm{d} \eta\right|_{\eta=0}$ vs. $\sqrt{s}$.

The charged particle pseudorapidity distributions at our two energies are shown in Fig. 2 , together with the UA5 data ${ }^{\mid 9]}$. The data, which are folded about $\eta=0$, all form plateaus over six units of $\eta$. The value of $\mathrm{dN} /\left.\mathrm{d} \eta\right|_{\eta=0}$ is $1.33 \pm .04$ times larger at $1.8 \mathrm{TeV}$ than at 630 
$\mathrm{GeV}$. The quoted error does not include the $10 \%$ systematic error common to both energies. The ratio of pleateau heights averaged over the range $|\eta|<1$ is, on a similar basis, 1.27 \pm .04. This will be used in the next section in deriving $\left\langle p_{T}\right\rangle$. These ratios are slightly higher than the value $1.22-1.25$ predicted by the Dual Parton Model ${ }^{[10]}$, or the value 1.20 predicted by the Lund-PYTHIA Monte Carlo program ${ }^{[11]}$.

Fig. 3 shows the values of $\mathrm{dN} /\left.\mathrm{d} \eta\right|_{\eta=0}$ as a function of $\sqrt{s}$. Good fits to the UA5 non-single-diffractive data and to the present CDF data (which are approximately an NSD sample) are obtained with either of the following s dependences:

\begin{tabular}{|cc|}
\hline $0.843 \mathrm{~s}^{0.102}$ & $\chi^{2}=5.4$ for 4 degrees of freedom \\
$1.129+0.0122 \ln ^{2}(\mathrm{~s})$ & $\chi^{2}=6.1$ for 4 DOF \\
\hline
\end{tabular}

The two fits, which are shown as the top curve, are almost indistinguishable, and differ by at most $1.5 \%$ over the range of the fit. The power law fit has essentially the same s dependence as the earlier fit to total inelastic data at lower energies. The plateau clearly rises faster than $\ln (\mathrm{s})$.

Fig. 4 shows charged particle multiplicity distributions in terms of the Koba-NielsenOlesen (KNO) variables $\left\langle N>\mathrm{P}(\mathrm{N})=\psi\left(\mathrm{N} /<N>\right.\right.$ ), where $\mathrm{P}(\mathrm{N})=\sigma(\mathrm{N}) / \sigma_{\text {inelastic. KNO }}$ scaling states that $\psi$ is independent of $\mathbf{s}$. Scaling works fairly well at low $\mathbf{s}$ and is clearly broken ${ }^{[12]}$ between ISR energies $(\sqrt{s} \sim 23-63 \mathrm{GeV})$ and $546 \mathrm{GeV}$. Fig. 5 shows CDF 1.8 TeV data (preliminary) and UA5 data at $546 \mathrm{GeV}$, where the charged particles are counted in progressively shorter ranges of $\eta$. The KNO distribution broadens substantially for smaller $\eta$ ranges, perhaps reflecting larger statistical fluctuations due to smaller track samples per event. The distributions are very similar at the two energies. The $1.8 \mathrm{TeV}$ data use uncorrected charged particle multiplicities. The systematics of high multiplicity events are still under study, and the inefficiencies could be relatively large.

\section{TRANSVERSE MOMENTUM DISTRIBUTIONS AND $<p_{T}>$}

Since this talk was presented, these results have appeared in print ${ }^{[13]}$, to which the reader is referred for details of the analysis and for some of the figures. The CTC has $>99 \%$ efficiency for tracks with $p_{T}>400 \mathrm{MeV} / \mathrm{c}$ and $|\eta|<1$. The charged particle invariant cross section is defined as:

$$
E d^{3} \sigma / d^{3} p=\left(\sigma_{e f f} / N_{e v t}\right)\left(N / p_{T} \cdot \Delta p_{T} \cdot \Delta \phi \cdot \Delta y\right)
$$

where $\mathrm{N}_{\text {evt }}$ is the number of events used and $\mathrm{N}$ is the number of charged tracks in a given element of phase space. The invariant cross section as a function of $p_{T}$ for the CDF 630 $\mathrm{GeV}$ data (which extends only to $3.5 \mathrm{GeV} / \mathrm{c}$ ) is in good agreement with the UA1 and UA2 data at $546 \mathrm{GeV}$. The CDF data at $1.8 \mathrm{TeV}$ extend to $p_{T}=10 \mathrm{GeV} / \mathrm{c}$, and fall less rapidly with $p_{T}$ than the $630 \mathrm{GeV}$ data ${ }^{|13|}$, being a factor of 4 higher at $3.5 \mathrm{GeV} / \mathrm{c}$. Invariant cross sections were fitted to a parton-model-inspired power law

$$
E d^{3} \sigma / d^{3} p=A \frac{p_{o}^{n}}{\left(p_{T}+p_{o}\right)^{n}}
$$

The parameters $\mathrm{p}_{0}$ and $\mathrm{n}$ are highly correlated and good fits can be obtained with a change in $n$ compensating a change in $\mathrm{p}_{o}$. We fix $\mathrm{p}_{o}=1.3 \mathrm{GeV} / \mathrm{c}$ as is done at lower $\sqrt{s}$. (When 
$\mathrm{p}_{o}$ is left free, the fit at $1.8 \mathrm{TeV}$ gives $\left.\mathrm{p}_{o}=1.29\right)^{[13]}$. Values of $\mathrm{n}$ decrease as the spectra harden with increasing s:

\begin{tabular}{|lrc|}
\hline detector & $\sqrt{s}$ & $\mathrm{n}$ \\
UA1 & $546 \mathrm{GeV}$ & $9.14 \pm .02$ \\
CDF & $630 \mathrm{GeV}$ & $8.89 \pm .06$ \\
CDF & $1.8 \mathrm{TeV}$ & $8.28 \pm .02$. \\
\hline
\end{tabular}

Fig. 5 shows the CDF $1.8 \mathrm{TeV}$ data, together with the UA1 data, ISR data (British Scandinavian) and Fermilab fixed target data (Chicago Princeton). ${ }^{[13]}$ The $1.8 \mathrm{TeV}$ data fall much less steeply with $p_{T}$, being 5 orders of magnitude larger than the $27 \mathrm{GeV}$ data at $p_{T}$ $=7 \mathrm{GeV} / \mathrm{c}$.

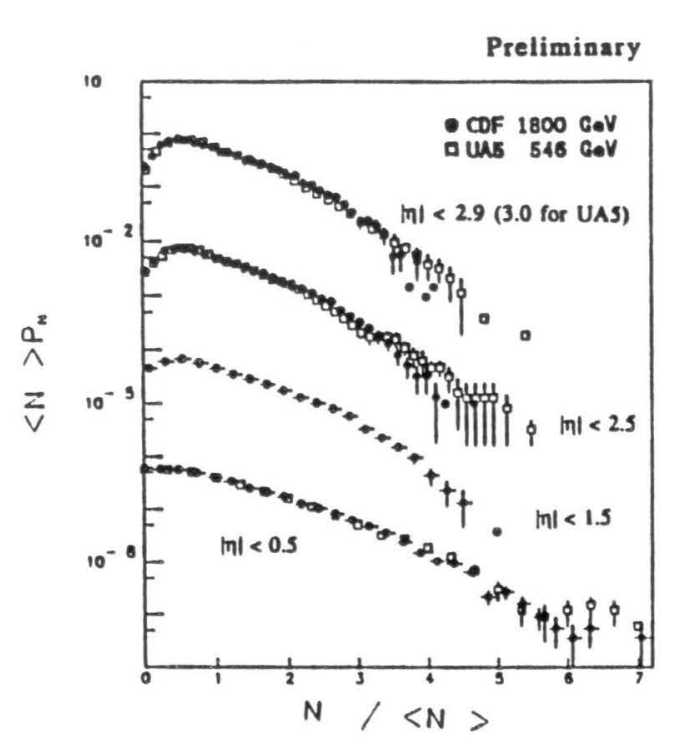

Fig. $4<N>P_{N}$ vs. $N /<N_{>}$,

KNO distributions for CDF $1.8 \mathrm{TeV}$ data and UA5 $546 \mathrm{GeV}$ data.

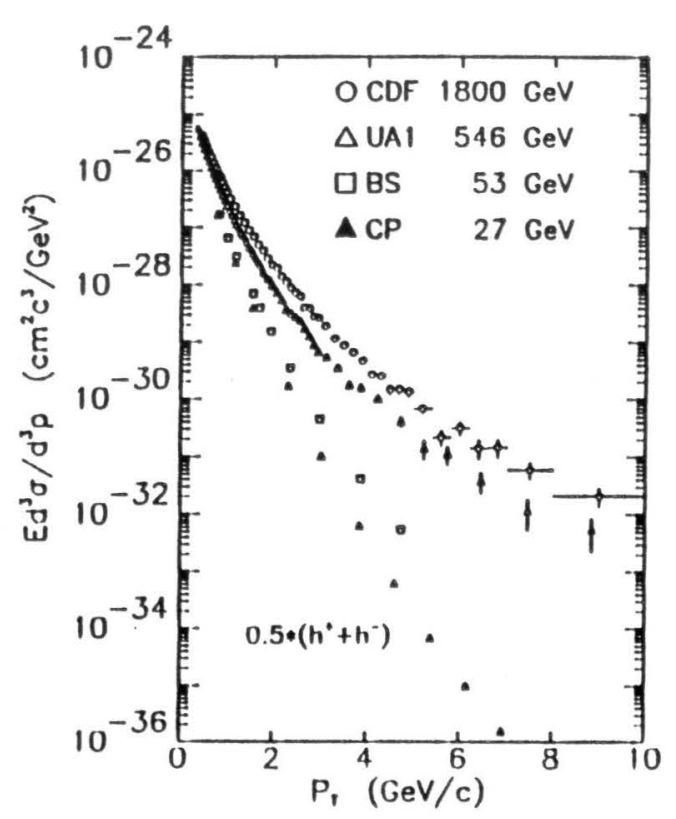

Fig. 5 Charged particle $\left(h^{+}+h^{-}\right) / 2$ invariant cross section vs. $p_{T}$.

A calculation by S. Ellis and J. Stirling ${ }^{|14|}$, based on the parton model and QCD, attempts to predict the above data. The ingredients are (a) structure functions $F\left(x, Q^{2}\right)$ for two colliding partons, one in the proton and one in the anti-proton determined from experiment and from QCD evolution in Feynman $x$, (b) fragmentation functions $D(z)$ for production of various hadrons, again determined from experiment, and (c) a QCD parton-parton scattering cross section $\hat{\sigma}(\hat{s}, \hat{t}, \hat{u})$. Fig. 6 shows the results of the calculations, using Duke and Owens set 1 structure functions with $\mathrm{Q}^{2}=p_{T}^{2}$ and a K-factor of 2 . Compared with the data of Fig. 5 above $p_{T}=1 \mathrm{GeV} / \mathrm{c}$, the $p_{T}$ and $\mathrm{s}$ dependences agree, within a factor of $\sim 2$ to 5 , over nine decades of invariant cross section.

In determining $\left\langle p_{T}>\right.$, extrapolation is required for the substantial portion of the $p_{T}$ 
spectrum below the $400 \mathrm{MeV} / \mathrm{c}$ çutoff. Alternative functional forms used include: (a) the above power law form, (b) a simple exponential $\mathrm{A} \exp \left(-\mathrm{b} p_{T}\right)$ and (c) a polynomial $\mathrm{A}\left(p_{T}^{2}+\right.$ B. $p_{T}+$ C). Systematic uncertainties due to choice of functional form are much reduced by the powerful constraint that the area under the entire curve must be given by $d N / d \eta$. When the three functions are fitted to the data between 400 and $600 \mathrm{MeV} / \mathrm{c},(\mathrm{Fig}$. 7) with $d N / d \eta$ fixed, the systematic error in the extrapolation is reduced to $0.003 \mathrm{GeV} / \mathrm{c}$. We use the well determined ratio $1.27 \pm .04$ of the plateau heights at 1.8 and $0.63 \mathrm{TeV}$ (from Section 5), and fix $d N / d \eta=3.30 \pm 0.15$ at $630 \mathrm{GeV}$ by interpolating measurements in the range 200 to $900 \mathrm{GeV}^{[9]}$. We then obtain $\left\langle p_{T}\right\rangle=0.495 \pm 0.014(0.432 \pm 0.004) \mathrm{GeV} / \mathrm{c}$ at $1.8(0.63)$ $\mathrm{TeV}$. There is an additional common uncertainty of $0.020 \mathrm{GeV} / \mathrm{c}$ on both numbers due to the $5 \%$ uncertainty on $d N / d \eta$ at $630 \mathrm{GeV}$. The $\mathrm{C} 0$ experiment at $1.8 \mathrm{TeV}$ quotes $\left\langle p_{T}\right\rangle=$ $0.47 \pm 0.01^{|15|}$. This value and the CDF value at $1.8 \mathrm{TeV}$ are in good statistical agreement.
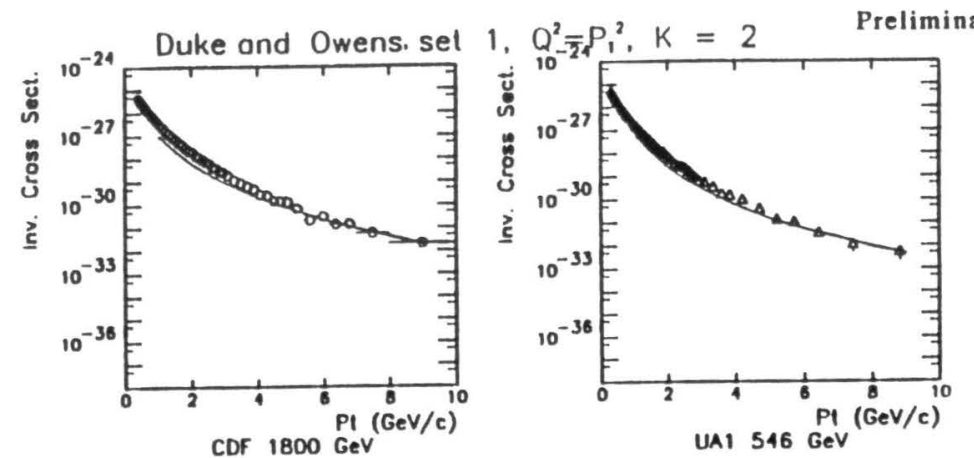

Fig. 6 Curves are a parton model calculation of Ellis and Stirling. Data are as in fig. 5.
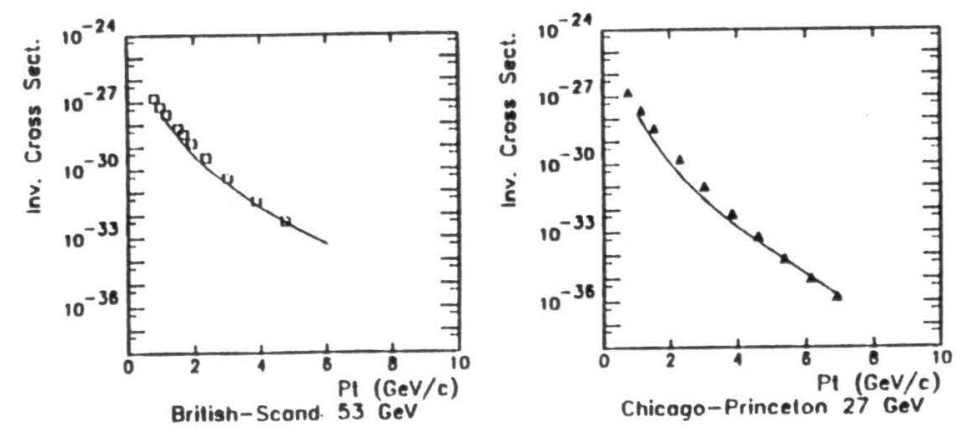

Fig. 7 Curves are (left to right) functional forms $a, b$, and $c$ described in the text, used to extrapolate to $p_{T}=0$.

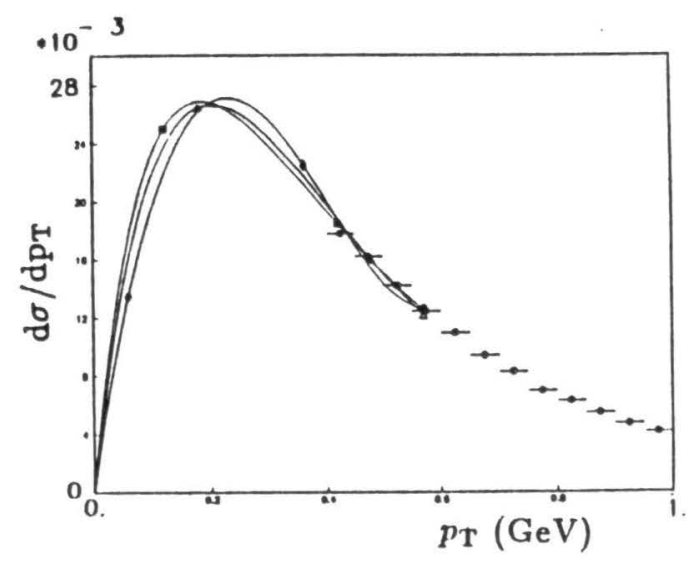


Fig. 8 shows $<p_{T}>$ as a function of $\mathrm{s}$ which rises substantially faster than $\ln (\mathrm{s})$. The common uncertainty of the two CUF points is omitted, and will not affect the steep slope from 0.63 to $1.8 \mathrm{TeV}$. The solid curve is a fit to the form $0.326+0.0044 \mathrm{~s}^{0.24}$, with $\chi^{2}=7.5$ for 9 degrees of freedom. The dashed curve is a fit to the form $0.433-0.025 \ln (\mathrm{s})+0.0019$ $\ln ^{2}(\mathrm{~s})$, with $\chi^{2}=9.35$. Either curve describes the data well, and it is clear that a function linear in $\ln (\mathrm{s})$ would give a very poor fit.

Fig. $8<p_{T}>$ as a function of $\sqrt{s}$. The two curves are fits described in the text.
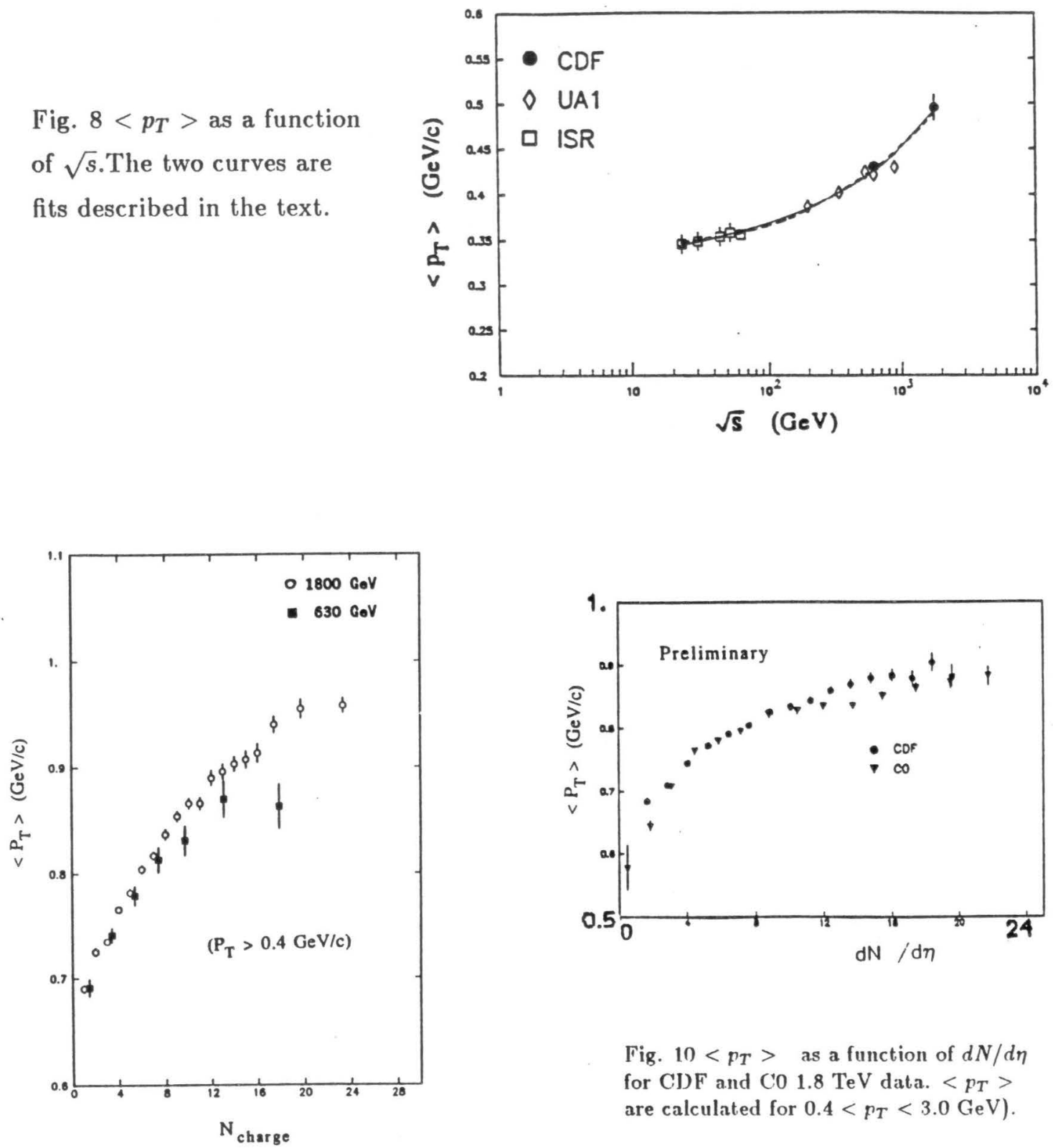

Fig. $9<p_{T}>$ (calculated for $\left.p_{T}>0.4 \mathrm{GeV}\right)$ vs. $N$ in the C'TC. Data at 1.8 and $0.63 \mathrm{TeV}$.

Fig. $\left.10<p_{T}\right\rangle$ as a function of $d N / d \eta$ for CJPF and $\mathrm{C} 01.8 \mathrm{TeV}$ data. $\left\langle p_{T}\right\rangle$ are calculated for $\left.0.4<p_{T}<3.0 \mathrm{GeV}\right)$. 
We have determined $<p_{T}>$ for various ranges of charged particle multiplicity seen in the range $|\eta|<1.0$ in the CTC (Fig. 9). The $d N / d \eta$ constraint is lost, and $\left\langle p_{T}\right\rangle$ is averaged over the range of the data from 0.4 to $10 \mathrm{GeV} / \mathrm{c}$, and no extrapolation is attempted. Within the statistical errors, the data are consistent with a smooth increase of $\left\langle p_{T}\right\rangle$ with increasing $\mathrm{N}$. There is no evidence for a phase transition as postulated for the Quark Gluon Plasma (QGP). However, in the absence of charged particle data below $400 \mathrm{MeV} / \mathrm{c}$, conclusions pro or con the QGP are not warranted. The $\mathrm{C} 0$ experiment has data down to $p_{T}=150 \mathrm{MeV} / \mathrm{c}^{[15]}$, and is complementary to $\mathrm{CDF}$ in the $p_{T}$ range covered.. Fig. 10 compares $\mathrm{CDF}$ and $\mathrm{C} 0$ data at $1.8 \mathrm{TeV}$, where $\left\langle p_{T}\right\rangle$ is averaged over the range $0.4<p_{T}<3.0 \mathrm{GeV} / \mathrm{c}$, common to both experiments. VTPC multiplicities for CDF are from $|\eta|<2.5$ (caveats of Section 5 apply). C'0 multiplicities are for $|\eta|<3.25$. The data are in reasonable agreement, and have rather smooth variation with $d N / d \eta$.

\section{7. $\mathrm{K}_{s}^{\circ}$ DATA}

Neutral strange particles decaying into 2 charged particles ("Vees") were studied using CTC tracks with momentum $p_{T}>0.25 \mathrm{GeV} / \mathrm{c}$ and having transverse impact parameter $\mathrm{d}$ $\geq 2 \mathrm{~mm}$ with respect to the primary event vertex. A geometrical fit to the Vee, with one degree of freedom, was accepted if the $\chi^{2}$ was less than 5 . The impact parameter of the Vee momentum vector was required to be: $\mathrm{D}_{\text {pair }}<2 \mathrm{~cm}$. To reduce background from primary charged particles, the Vee vertex was required to be at a radius $\mathrm{R}>2 \mathrm{~cm}$.

A search for $\mathrm{K}_{g}^{o}$ in the $1.8 \mathrm{TeV}$ data yielded 402 events above a background of 64 events, as seen in the $\pi^{+} \pi^{-}$invariant mass plot (Fig. 11). A fit gives a mass $\mathrm{M}=496.8 \mathrm{MeV} / \mathrm{c}^{2}$, a mass resolution $\sigma_{M}=9.2 \mathrm{MeV} / \mathrm{c}^{2}$, and an almost linear background (with a very small term quadratic in $\mathrm{M}$ ), with $\chi^{2}=$ for degrees of freedom. Events neighboring the $\mathrm{K}_{s}^{0}$ mass peak, representing the background in the signal region, were subtracted for all $\mathrm{K}_{s}^{0}$ distributions.

Acceptance and track- and Vee- finding efficiencies were determined by implantation into real events of Monte Carlo $\mathrm{K}_{s}^{o}$, generated with full CDF detector simulaton. The overall efficiency, which was used to weight each event, is between 0.14 and 0.28 for $p_{T}$ in the range 1.4 to $10 \mathrm{GeV} / \mathrm{c}$. The efficiency becomes very small below $600 \mathrm{MeV} / \mathrm{c}$, due to spiralling in the $\mathrm{CTC}$ of tracks below $330 \mathrm{MeV} / \mathrm{c}$ and due to the applied track momentum cut at $250 \mathrm{MeV} / \mathrm{c}$. The $\mathrm{K}_{s}^{o}$ efficiency has recently been found to depend on the charged particle multiplicity in the CTC. Extensive MC implantation studies are underway, and the results may substantially affect the $\mathrm{K}_{s}^{o}$ distributions.

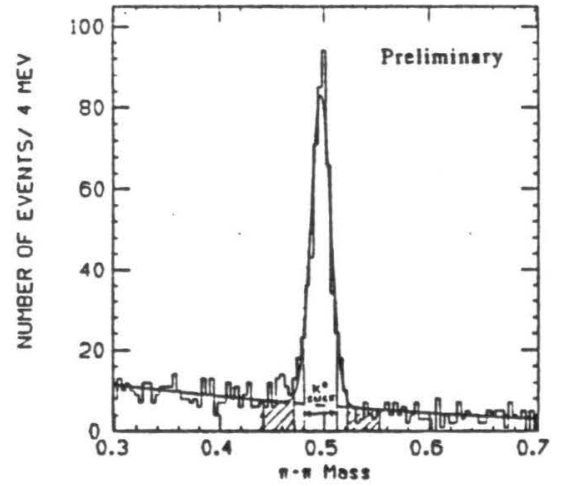

Fig. 11 Vee $\rightarrow \pi^{+} \pi^{-}$effective mass plot, with Gaussian fit to $K_{n}^{0}$, as described in text.

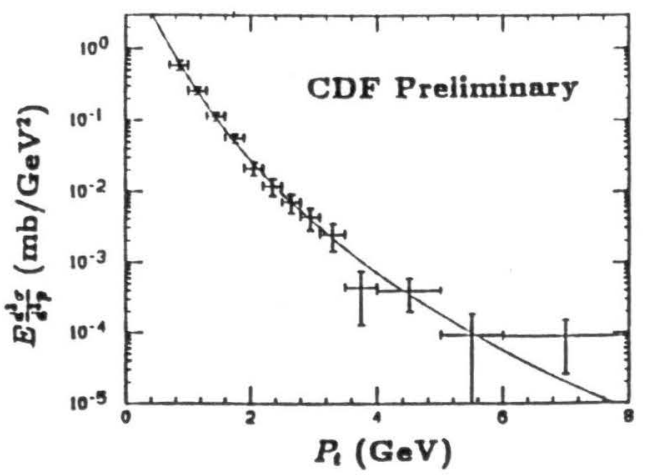

Fig. $12 K_{a}^{0}$ invariant cross section as a function of $p_{T}$, for CDF $1.8 \mathrm{TeV}$ data.

A preliminary $\mathrm{K}_{s}^{o}$ invariant cross section as a function of $p_{T}$ is shown in Fig. 12. It 
was fitted over the range $0.7 \leq p_{T} \leq 8.0 \mathrm{GeV} / \mathrm{c}$, using a Poisson likelihood function, to the same power law form as for the charged hadron spectrum, again with $p_{o}=1.3 \mathrm{GeV} / \mathrm{c}$. The exponent is found to be $\mathrm{n}=7.49 \pm 0.23$. It is smaller than for the charged hadron data by $\Delta \mathrm{n}=0.79 \pm 0.23$, corresponding to a ratio $\mathrm{K}_{s}^{0} / \mathrm{h}^{ \pm}$which rises with $p_{T}$. This preliminary value of $\mathrm{n}$ is consistent with values measured by $\mathrm{U} \Lambda 5$ at lower $\sqrt{s}^{|16|}$, for example $\mathrm{n}=7.57$ \pm 0.39 at $900 \mathrm{GeV}$. It is also consistent with a continuing decrease of $\mathrm{n}$ as $\sqrt{s}$ increases ${ }^{|16|}$, reflecting a continued hardening of the $\mathrm{K}^{\circ} \mathrm{s} p_{T}$ spectrum.

\section{CHARGED PARTICLE CORRELATIONS}

A tendency is seen for charged tracks to cluster in $\eta$ and $\phi$. The CTC track sample studied is restricted to $|\eta|<1.0$. Fig. 13 shows the charged particle transverse momentum density around trigger particles having various ranges of $p_{T}$. The distributions exclude the $p_{T}$ of the trigger particle. Fig. 13a shows $\mathrm{d} \sigma_{p_{T}} / \mathrm{d} \Delta \eta$ as a function of distance in pseudorapdity from the trigger particle, for "same-side" tracks having $\phi$ within $90^{\circ}$ of the trigger particle. There is clear peaking around the trigger particle, which becomes more pronounced for larger trigger $p_{T}$. Fig. 13b shows a strong tendency for momentum density $\mathrm{d} \sigma_{p_{T}} / \mathrm{d} \Delta \phi$ to peak both in the direction of the trigger particle and also at $\mathrm{d} \phi=180^{\circ}$ opposite to the trigger particle. Fig. 13c is for away-side tracks, in the $\phi$ semicircle opposite to the trigger particle. No $\eta$ correlation is seen.

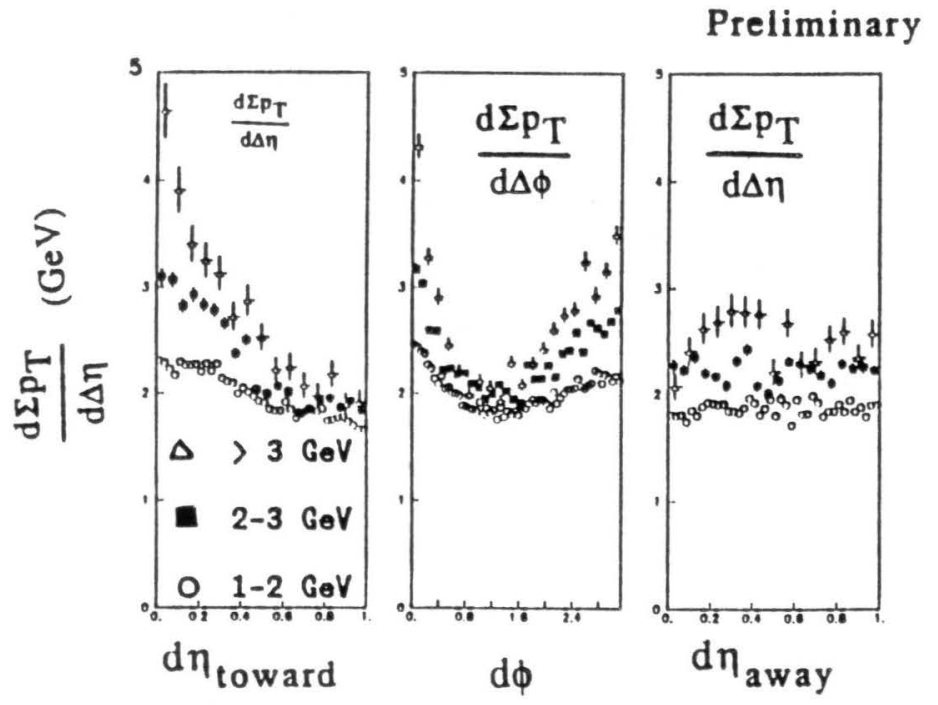

Fig. 13 Charged particle momentum densities around trigger particles of various $p_{T}$, for (a) same-side and (c) away-side particles as a function of distance in $\eta$ from trigger particle; (b) as a function of distance in $\phi$ from trigger particle.

The same patterns are seen, but are much more pronounced, in a sample of jet events (not shown ) which were selected using a central calorimeter jet trigger with $70 \mathrm{GeV}$ threshold and which is dominated by two-jet events. The absence (presence) of away-side correlations in $\eta(\phi)$ can be understood in terms of parton-parton collisions with small overall $p_{T}$ and conservation of $p_{T}$, and with a wide spread of overall longitudinal momentum, $p_{Z}$, reflecting the Feynman-x distributions of the colliding partons.

The patterns of Fig. 13 are consistent with the observation of the charged portion of low- $p_{T}$ jets. This further motivates a search for charged clusters using an algorithm similar 
to the calorimeter transverse energy $E_{T}$ clustering algorithm used to find jets ${ }^{[5]}$. Using only charged tracks has certain advantages over calorimeter clustering for low $p_{T}$ jets: (a) track momentum resolution $\sigma_{p_{T}} / p_{T}$ is less than $2.5 \%$ for the MinBias event sample, (b) track directions at the primary vertex are used, whereas low momentum tracks are swept by the magnetic field to the wrong azimuth at the calorimeter, (c) the calorimeter response is known to be nonlinear at low $E_{T}$. Disadvantages of clustering charged tracks include: (a) on average, one-third or more of the hadronic energy is expected to be in neutral particles and (b) event-to-event fluctuations in the neutral to charged energy ratio will smear the jet energy estimate.

We use the CTC track sample with $|\eta|<1.0$. A charged cluster is defined as a set of tracks within a cone of half-angle $40^{\circ}$ around a common axis along the cluster momentum vector. Only tracks having rapidity $\mathrm{y}>\mathrm{y}_{o}=2.0$ along the common cone axis are used. 'The search starts with the axis along the highest- $p_{T}$ track, and iteratively adjusts the cone axis as the cluster is formed. Particles are used in descending $p_{T}$ until up to 4 clusters are found. The cluster transverse energy is then calculated:

$$
E_{T}^{c l u s t}=\left[\left(\Sigma E_{i}\right)^{2}-\left(\Sigma P_{z i}\right)^{2}\right]^{1 / 2}
$$

where the $\mathrm{E}_{i}$ are calculated using the pion mass.

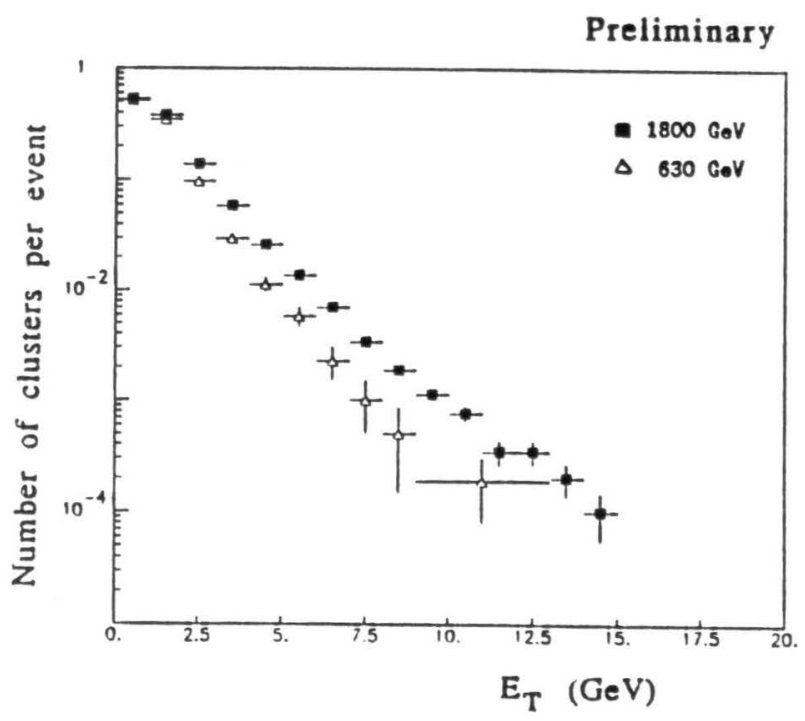

Fig. $14 E_{T}$ distribution of charged clusters for CDF data at $1.8 \mathrm{TeV}$ and $630 \mathrm{GeV}$.

The $E_{T}^{\text {clust }}$ spectra for clusters with $|\eta|<0.7$ are shown in Fig. 14 for $1.8 \mathrm{TeV}$ and 630 $\mathrm{GeV}$ MinBias events. The spectrum is seen to harden as $\sqrt{s}$ increases, with a factor of 4 rise for $E_{T}^{\text {clust }} \sim 7$ to $10 \mathrm{GeV}$. These charged clusters occur quite copiously, with 35 to $40 \%$ probability per MinBias event for $1 \leq E_{T}^{\text {clust }} \leq 2 \mathrm{GeV}$. At 1.8 ' leV the integrated probability of a cluster with $E_{T}^{\text {clust }}>3 \mathrm{GeV}$ is $11 \%$ per event, corresponding to a preliminary cross section of $4.7 \mathrm{mb}$ in the central 1.4 units of $\eta$. 


\section{ACKNOWLEDGEMENTS}

The CDF collaboration wishes to thank the CDF technical support staff, and the staff of the TeVatron Collider, for their invaluable contributions to this experiment. This work was supported by the U.S. Department of Energy, the National Scoience Foundation, the Italian Instituto Nazionale di Fisica Nucleare, the Japanese Ministry of Science, Culture and Education, and the A. P. Sloan Foundation.

\section{REFERENCES}

1) F. Abe et al., Nucl. Inst. Meth. A271 (1988) 387.

2) G. Altarelli, K. Ellis, M. Greco, and G. Martinelli, Nucl. Phys. B246 (1984) 12;

G. Altarelli, K. Ellis, and G. Martinelli, Z. Phys. C27 (1985) 617.

3) F. Abe et al., A Measurement of $\mathrm{W}$ Boson Production in $1.8 \mathrm{TeV}$ pp Collisions, (submitted to Phys. Rev. Lett.).

4) E. Eichten, Theoretical Expectations at Collider Energies, Fermilab-Conf-85/178-T (Fermilab report, May, 1986) p. 92.

5) F. Abe et al., Measurement of the Inclusive Jet Cross Section at the Tevatron p̄p Collider, (submitted to Phys. Rev. Lett.).

6) F. Snider et al., Nucl. Inst. Meth. A268 (1988) 75.

7) F. Bedeschi et al., Nucl. Inst. Meth. A268 (1988) 50.

8) D. Bernard et al., Phys. Lett. 186B (1987) 227.

9) G. Ekspong, Nucl. Phys. A461 (1987) 145, and references therein.

10) Dual Parton Model, A. Capella, U. Sukhatme, C-I Tan, and J. Tran Thanh Van, Multiparticle Production, ed. P. Carruthers (World Scientific,1988); ratio is from a Monte Carlo program made available by J. Tran Thanh Van. For an extension of the DPM to include hard parton collisions, see J. Ranft et al., SSC Central Design Group report SSC-149 (December, 1987); ratio is from a Monte Carlo calculation by J. Ranft (private communication).

11) H-U Bengtsson and T. Sjostrand, Computer Phys. Communications 46 (1987) 43.

12) G. J. Alner et al., Phys. Lett. 138B (1984) 304, G. J. Alner et al., Phys. Lett. 160B (1984) 199.

13) F. Abe et al., Phys. Rev. Lett. 61 (1988) 1819.

14) S. Ellis and W. J. Stirling, private communication.

15) C. Lindsay, Recent Results from E-735: Search for Quark Gluon Plasma in p̄p Collisions at $\sqrt{s}=1.8 \mathrm{TeV}$, this volume.

16) R. Ansorge et al., Phys. Lett. B199 (1987) 311, and submitted to Zeit. Phys. C. 